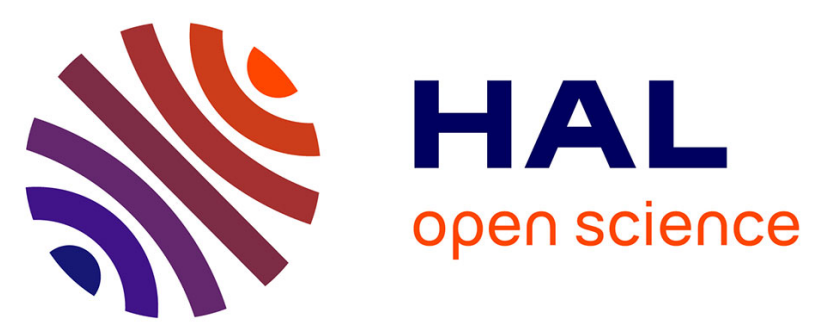

\title{
Conventional versus stealth lipid nanoparticles: formulation and in vivo fate prediction through FRET monitoring.
}

Anne-Laure Lainé, Julien Gravier, Maxime Henry, Lucie Sancey, Jérôme Bejaud, E Pancani, M Wiber, Isabelle Texier, Jean-Luc Coll, Jean-Pierre

Benoit, et al.

\section{To cite this version:}

Anne-Laure Lainé, Julien Gravier, Maxime Henry, Lucie Sancey, Jérôme Bejaud, et al.. Conventional versus stealth lipid nanoparticles: formulation and in vivo fate prediction through FRET monitoring.. Journal of controlled release: official journal of the Controlled Release Society, 2014, 188, pp.1-8. 10.1016/j.jconrel.2014.05.042 . hal-03179480

\section{HAL Id: hal-03179480 \\ https://univ-angers.hal.science/hal-03179480}

Submitted on 24 Mar 2021

HAL is a multi-disciplinary open access archive for the deposit and dissemination of scientific research documents, whether they are published or not. The documents may come from teaching and research institutions in France or abroad, or from public or private research centers.
L'archive ouverte pluridisciplinaire $\mathbf{H A L}$, est destinée au dépôt et à la diffusion de documents scientifiques de niveau recherche, publiés ou non, émanant des établissements d'enseignement et de recherche français ou étrangers, des laboratoires publics ou privés. 


\title{
Conventional versus stealth lipid nanoparticles: Formulation and in vivo fate prediction through FRET monitoring
}

\author{
A.-L. Lainé ${ }^{\mathrm{a}, \mathrm{b}}$, J. Gravier ${ }^{\text {c,d,e }}$, M. Henry ${ }^{\mathrm{c}, \mathrm{d}}$, L. Sancey ${ }^{\text {c,d }}$, J. Béjaud ${ }^{\mathrm{a}, \mathrm{b}}$, E. Pancani ${ }^{\mathrm{a}, \mathrm{b}}$, \\ M. Wiber ${ }^{\mathrm{a}, \mathrm{b}}$, I. Texier ${ }^{\mathrm{e}}$, J.-L. Coll ${ }^{\mathrm{c}, \mathrm{d}}$, J.-P. Benoit ${ }^{\mathrm{a}, \mathrm{b}}$, C. Passirani ${ }^{\mathrm{a}, \mathrm{b}, *}$ \\ a LUNAM Université - Micro et Nanomédecines Biomimétiques, F-49933 Angers, France \\ b INSERM U1066, IBS-CHU, 4 Rue Larrey, F-49933 Angers Cedex 9, France \\ ' INSERM U823, Institut Albert Bonniot, F-38 706 Grenoble, France \\ d Université Joseph Fourier, F-38706 Grenoble, France \\ e CEA, LETI, DTBS, 17 Avenue des Martyrs, F-38054 Grenoble Cedex 9, France
}

\section{A R T I C L E I N F O}

Article history:

Received 11 February 2014

Accepted 20 May 2014

Available online 28 May 2014

\section{Keywords:}

Biodistribution

Fluorescence imaging

Nanomedicine

Encapsulation stability

Drug release

\begin{abstract}
A B S T R A C T
The determination of the nanocarrier fate in preclinical models is required before any translation from laboratory to clinical trials. Modern fluorescent imaging techniques have gained considerable advances becoming a powerful technology for non-invasive visualization in living subjects. Among them, Forster (fluorescence) resonance energy transfer (FRET) is a particular fluorescence imaging which involves energy transfer between 2 fluorophores in a distance-dependent manner. Considering this feature, the encapsulation of an acceptor/donor pair in lipid nanoparticles (LNEs: lipid nanoemulsions, LNCs: lipid nanocapsules) allowed the carrier integrity to be tracked. Accordingly, we used this FRET technique to evaluate the behavior of LNEs, conventional LNCs and newly designed stealth LNCs. After the development through a one-step (OS) PEGylation process of these stealth LNCs (OS LNCs), in vitro guest exchange dynamics and release kinetics were evaluated for both LNC formulations. We thereafter assessed in vivo biodistribution of all types of lipid nanoparticles. Results showed enhanced stability of encapsulation in OS LNCs in comparison to conventional LNCs. Additionally, the presence of the long PEG chains on the lipid nanoparticle surface altered the biodistribution pattern. Despite different release kinetic profiles, OS LNCs and LNEs showed extended blood circulation time associated with a good structure stability over several hours after intravenous injection.
\end{abstract}

(C) 2014 Elsevier B.V. All rights reserved.

\section{Introduction}

Lipid nanoparticles have been taking an active part in the nanomedicine landscape for the past decade. Their fairly simple preparation process is based on the well-known phase inversion temperature method of an emulsion (lipid nanocapsules, LNCs) or on ultrasonication (lipid nanoemulsions, LNEs). LNCs and LNEs are structured as an oily core composed of medium (LNCs) or long (LNEs) chain triglycerides, and surrounded by a surfactant shell made of a polyethylene glycol based surfactant and phospholipids. A comprehensive study of the ternary diagram permitted to define nanoparticle feasibility area and settle a precise ratio between excipients. Accordingly, the particle size is tunable depending on the composition allowing the achievement of stable and monodisperse nanoparticles ranging from 20 to $100 \mathrm{~nm}$ [1-3]. Lipid nanoemulsions have been used to design performing imaging agents for tumor and lymph node imaging [4-7]. Lipid nanocapsules have represented a great tool to develop injectable dosage forms for

\footnotetext{
* Corresponding author at: INSERM U1066, IBS-CHU, 4 Rue Larrey, 49933 Angers Cedex 9, France. Tel.: + 332446885 34; fax: + 33244688546 .

E-mail address: Catherine.passirani@univ-angers.fr (C. Passirani).
}

lipophilic drugs and have already shown promising vectorization ability for various anticancer drugs including paclitaxel [8], docetaxel [9], rhenium complexes [10], metal-based drugs [11] and polyphenols [12]. Firstly developed as hydrophobic drug delivery systems, they can also entrap hydrophilic agents such as doxorubicin [13] and nucleic acids as DNA [14] and siRNA [15].

Today, LNCs and LNEs have reached an advanced level in terms of preparation as well as characterization and showed promising performance as drug delivery systems. With an organic solvent free and low energy consumer process and composed of only FDA-approved excipients, they meet all the requirements to make clinically relevant nanocarriers. However, the translation to the clinic can only be considered if the nano-object fate in vivo is perfectly mastered including biodistribution, interactions with biological barriers, elimination and toxicity profile.

Whereas in vivo distribution of inorganic nanoparticles such as gold nanoparticles, iron oxide nanoparticles and carbon nanotubes can be easily monitored thanks to their imaging properties, tracking organic nanocarriers faces some difficulties. Common methods used to assess the biodistribution of nanocarriers involve a radionuclide, fluorescent or magnetic agent while the labeled element can be either a particle 
component or an entrapped probe. However, the pharmacokinetic (PK) profile obtained through those methods is highly and solely dependent on the distribution and elimination profile of the tracked element. Consequently, depending on the tracers used, it can conduct to discrepancy according to the metabolization pathway of the probe. This phenomenon was observed with LNCs and LNEs. Various techniques have been used over the years to evaluate their biodistribution. Ballot et al. used a radiolabeled complex composed of Technetium-99m or Rhenium188 encapsulated into LNCs to monitor the biodistribution and pharmacokinetic parameters of the carrier [16]. Similar PK profiles were obtained for both radionuclides with a half-life of around $20 \mathrm{~min}$. Few years later, Lacoeuille et al. performed biodistribution studies through the radiolabeling of either the oily core with ${ }^{14} \mathrm{C}$-trimyristin or the shell with ${ }^{14} \mathrm{C}$-phosphatidylcholine resulting in a $\mathrm{t}_{1 / 2}$ of $2.4 \mathrm{~h}$ and $2.9 \mathrm{~h}$ respectively [8]. More recently, a PK study was carried out through fluorescence analysis by the encapsulation of the Dil probe into LNCS [17]. The $t_{1 / 2}$ achieved by these DiI-LNCs was $2 \mathrm{~h}$. As for the LNEs, Merian et al. studied their in vivo fate using triply labeled nanoparticles (DiD dye, cholesteryl $-{ }^{14} \mathrm{C}$-oleate, ${ }^{3} \mathrm{H}$-cholesteryl-hexadecyl-ether) [18]. Similar PK profiles were obtained for the two radiotracers over the first $8 \mathrm{~h}$ post-injection, with a half-life of around $30 \mathrm{~min}$, but elimination phases were different. The various PK parameters obtained with these diverse tracers highlight a tracer-dependent tracking not representative of the particle distribution pattern. More importantly, these nanoparticles are designed to be biodegradable once they have reached their site of action, but information on their degradation kinetics and associated drug release are missing. In light of these considerations, none of the previously used techniques was able to appropriately track the entire lipid nanostructure and their in vivo fate.

Modern fluorescent imaging has gained considerable advances becoming a powerful technology for non-invasive visualization in living subjects. Fluorescent tool is commonly used in rodents but also in clinics for image-guided surgery. Forster (fluorescence) resonance energy transfer (FRET) is a particular fluorescence imaging which involves energy transfer between 2 fluorophores, from an excited donor to an acceptor molecule. Since the energy transfer is highly dependent on the distance between the donor and acceptor and can only occur in the $1-10 \mathrm{~nm}$ range, this technique is widely used for biological phenomenon investigation such as protein conformational change, protein interaction and enzyme activity [19]. This distance-dependent FRET signal makes it a suitable technique to monitor nanoparticle integrity through the encapsulation of FRET pairs. For instance, this technique has been recently used to monitor the stability of polymeric nanoparticles in serum [20] as well as the interaction with the cell membrane [21]. However, in vivo imaging can be more challenging due to strong tissue auto-fluorescence and weak photon penetration in the living tissue. In order to overcome these issues, near infrared (NIR) fluorescent probes should be used as recently performed for in vivo imaging of nanoparticles [22,23].

For cancer therapy, nanocarriers are often endowed with stealth properties in order to promote the Enhanced Permeability and Retention (EPR) effect and ultimately increase drug accumulation in the cancer site. Indeed, in order to reduce the recognition by the mononuclear phagocyte system, nanocarrier PEGylation is often applied preventing protein adsorption and leading to extended circulation time. Consequently, such stealth lipid nanoparticles might be more relevant for clinical transfer. As conventional LNCs are covered only with short PEG chains (660 MW), their stealth properties can be improved by inserting longer PEG based molecules (2000 MW). Whereas these stealth LNCs were previously obtained through a post-insertion (PI) technique [24], we designed in this work an alternative PEGylation approach based on a one-step (OS) process permitting to simplify the whole formulation phase. Similarly to stealth LNCs, LNEs are originally composed of long PEG chains in their surfactant shell (2000 MW).

In order to compare the stability of encapsulation in conventional LNCs and stealth LNCs, guest exchange dynamics and release kinetics were followed by FRET fluorescence. We assessed thereafter the in vivo biodistribution and stability of all types of FRET nanoparticles.

\section{Material and methods}

\subsection{Chemical materials}

The lipophilic Labrafac ${ }^{\circ}$ WL1349 (caprylic-capric acid triglycerides) and Suppocire NB ${ }^{\mathrm{TM}}$ were purchased from Gattefosse S.A. (Saint-Priest, France). Myrj ${ }^{\mathrm{TM}} 53$ (PEG 40 stearate, $1980 \mathrm{Da}$ ) and Super Refined Soybean Oil were obtained from Croda Uniqema (Chocques, France). Lipoïd® S753 (soybean lecithin at $69 \%$ of phosphatidylcholine) was provided by 2Lipoïd Gmbh (Ludwigshafen, Germany); Solutol® HS15 (a mixture of free polyethylene glycol 660 and polyethylene glycol 660 hydroxystearate) by BASF (Ludwigshafen, Germany) and $\mathrm{NaCl}$ by Prolabo (Fontenay-sous-bois, France). Deionized water was acquired from a Milli-Q plus system (Millipore, Paris, France) and sterile water from Cooper (Melun, France). 1,2-Distearoyl-sn-glycero-3-phosphoethanolamineN-[methoxy-(polyethyleneglycol)2000] (DSPE-mPEG2000) (Mean Molecular Weight $(\mathrm{MW})=2805 \mathrm{~g} / \mathrm{mol}$ ) was purchased from Avanti Polar Lipids (Alabaster, USA). Octadecylamine was acquired from Sigma. DiD (1,1'-Dioctadecyl-3,3,3',3'-tetramethylindodicarbocyanine perchlorate) and FP730-NHS were obtained from Invitrogen (Cergy, Pontoise, France) and from Interchim (Montluçon, France), respectively.

\subsection{Animals}

In vivo experiments were carried out on female NMRI nude mice (6-8 weeks old, Janvier, Le Genest-Saint-Isle, France). Animal care was performed in strict accordance with French Ministry of Agriculture regulations.

\subsection{Synthesis of FP730-C18}

$4 \mathrm{mg}$ of FP730-NHS was dissolved in $3 \mathrm{~mL}$ of $\mathrm{MeOH}$ (Fisher) and added with $2.85 \mathrm{mg}$ octadecylamine (2 eq). The reaction mixture was stirred in the dark at room temperature overnight, and the reaction advancement was monitored by thin layer chromatography. The final product FP730-C18 was purified on a silica gel column chromatography. A mixture of $\mathrm{CH}_{2} \mathrm{Cl}_{2} / \mathrm{MeOH}$ was used as the mobile phase through a gradient elution from $100 \% \mathrm{CH}_{2} \mathrm{Cl}_{2}$ (Fisher) to $80 / 20 \mathrm{CH}_{2} \mathrm{Cl}_{2} / \mathrm{MeOH}$.

\subsection{Nanoparticle formulation}

\subsubsection{FRET LNE formulation}

$50 \mathrm{~nm}$ diameter LNEs were prepared by the method described by Gravier et al. [6]. Appropriate dye amounts in $\mathrm{CH}_{2} \mathrm{Cl}_{2}(202 \mu \mathrm{g}$ of DiD, $522 \mu \mathrm{g}$ of FP730-C18, DiD:FP730-C18 molar ratio of 1:2.5) were poured into a $5 \mathrm{~mL}$ vial. The solvent was evaporated under vacuum before the oil premix (22.7 mg Soybean oil, $68 \mathrm{mg}$ Suppocire $\mathrm{NB}^{\mathrm{TM}}$ and $17.3 \mathrm{mg}$ Lipoid $\circledast$ ) was added. $92 \mathrm{mg}$ Myrj ${ }^{\mathrm{TM}} 53$ and $500 \mathrm{mg}$ of glycerol were dissolved in $154 \mathrm{mM} \mathrm{NaCl}$ aqueous solution (qsp $2 \mathrm{~mL}$ ) and added to the oily premix. The mixture was placed in a $60{ }^{\circ} \mathrm{C}$ water bath and sonicated for 5 min using a VCX750 Ultrasonic processor (power output $190 \mathrm{~W}$, probe diameter $3 \mathrm{~mm}$, Sonics, Newtown, USA). LNEs were dialyzed overnight at room temperature against $1 \times$ PBS buffer (12-14 kDa molecular weight cut off membranes, ZelluTrans, Roth, France) and passed through $0.2 \mu \mathrm{m}$ filters.

\subsubsection{Conventional LNC formulation}

LNCs were prepared according to a phase inversion temperature method as previously described [2]. Briefly, the preparation process consisted of mixing all the excipients (Solutol ${ }^{\circledR}$ HS15 $(16.9 \% \mathrm{w} / \mathrm{w})$, Lipoid $\AA(1.5 \% \mathrm{w} / \mathrm{w})$, Labrafac $₫(20.6 \% \mathrm{w} / \mathrm{w}), \mathrm{NaCl}(1.8 \% \mathrm{w} / \mathrm{w})$ and water $(59.2 \% \mathrm{w} / \mathrm{w}))$ under magnetic stirring. Three to four cycles of progressive heating and cooling between 90 and $60{ }^{\circ} \mathrm{C}$ were then 
carried out. At the last cooling step, the LNC suspension was diluted with water $(71.4 \% \mathrm{w} / \mathrm{w})$.

\subsubsection{Design of a "one-step (OS)" stealth LNC process}

In order to evaluate the feasibility of generating OS nanocapsules via the phase inversion method, PEG-based phospholipids (DSPE-mPEG) were added to the LNC mixture in various concentrations ranging from $0.1 \%$ to $8.1 \%\left(\mathrm{~W}_{\text {DSPE-mPEG }} / \mathrm{w}_{[\text {Solutol }}{ }^{\circledR}+\right.$ Lipoid ${ }^{\circledR}+$ Labrafac ${ }^{\circledR}+$ DSPE-mPEG $\left.]\right)$.

\subsubsection{FRET LNCS}

A hydrophobic FRET pair, DiD (donor) and FP730-C18 (acceptor) was used for this purpose. FRET LNCs were obtained by labeling the LNCs with the DiD probe at $1.2 \mathrm{mmol} / \mathrm{L}_{\text {Labrafac }}$. Fluorescent stealth LNCs were achieved by adding DiD and DSPE-mPEG 2000 (1,2-Distearoyl-snglycero-3-phosphoethanolamine- $\mathrm{N}$-[methoxy-(polyethyleneglycol)2000 ], $4.8 \% \mathrm{w} / \mathrm{w}$ ) in the LNC mixture. The amount of dilution water added at the last cooling step was reduced to $28.6 \% \mathrm{v} / \mathrm{v}$ in order to be able to perform the FP730-C18 encapsulation thereafter.

Then, FP730-C18 (3 mmol/L $\mathrm{L}_{\text {Labrafac }}, 2.5 / 1 \mathrm{DiD}$ ) was incorporated to DiD-LNCs and stealth DiD-LNCs by overnight stirring at room temperature and protected from light. The resulting FRET LNCs were purified through a dialysis against water for $8 \mathrm{~h}$ using a Spectra/Por ${ }^{\circledR}$ dialysis bag with MWCO of 15,000 Da. The resulting suspension was diluted in water $(42.8 \% \mathrm{v} / \mathrm{v})$ and passed through a $0.2 \mu \mathrm{M}$ filter.

\subsection{Nanoparticle characterization}

The average hydrodynamic diameter and the zeta potential of nanocapsules were determined at $25^{\circ} \mathrm{C}$, in triplicate, using a Malvern Zetasizer ${ }^{\circledR}$ (Nano Serie DTS 1060, Malvern Instruments S.A., Worcestershire, UK). For the measurement, the lipid nanoparticles were diluted in Milli-Q water ( $50 \mu \mathrm{L}$ dispersion in $2.95 \mathrm{~mL}$ water). All comparison experiments were carried out with similar conductivity values.

\subsection{Physico-chemical stability studies}

The stability of LNEs, conventional LNCs and OS LNCs (DSPE-mPEG = $4.8 \% \mathrm{w} / \mathrm{w}$ ) was assessed at $4{ }^{\circ} \mathrm{C}$ (storage condition) and $37{ }^{\circ} \mathrm{C}$ (physiological temperature). Each formulation was done in triplicate and split into two parts; one was stored at $4{ }^{\circ} \mathrm{C}$ and the second one at $37{ }^{\circ} \mathrm{C}$. The stability study was carried out over 28 days via zeta potential and size measurements.

The physical stability in human plasma of the various LNC types was also assessed. Blood samples were collected from the same consenting donor and incubated with EDTA. Plasma (provided by the Etablissement Français du Sang, CHU, Angers, France) was recovered as the supernatant of 2 consecutive centrifugation steps performed at $2000 \mathrm{~g}$ (10 min then $15 \mathrm{~min}$ ), and used immediately. For this purpose, $170 \mu \mathrm{L}$ of LNCs or LNEs was diluted into $2.83 \mathrm{~mL}$ of plasma (equivalent to in vivo dilution) and put into a shaking water bath with a temperature control set at $37^{\circ} \mathrm{C}$. Samples $(10 \mu \mathrm{L})$ were withdrawn at $1 \mathrm{~h}, 2 \mathrm{~h}, 4 \mathrm{~h}, 6 \mathrm{~h}, 24 \mathrm{~h}$ up to 5 days, diluted in water $(990 \mu \mathrm{L})$ and the whole suspension was passed through a $0.22 \mu \mathrm{M}$ filter in order to remove large proteins that could hamper the size measurement.

\subsection{Studies of FRET stability and exchange dynamics}

For the FRET stability studies, conventional FRET LNCs or FRET OS LNCs were diluted at $1 / 200$ in FBS (Lonza) and kept at $37{ }^{\circ} \mathrm{C}$ in a moving plate for $24 \mathrm{~h}$. Emission spectra were recorded at $1 \mathrm{~h}, 3 \mathrm{~h}, 5 \mathrm{~h}$ and $24 \mathrm{~h}$ using a Perkin-Elmer LS50B fluorimeter. The excitation wavelength was set at $620 \mathrm{~nm}$.

For the guest exchange dynamic studies, DiD and FP730-C18 were independently encapsulated into conventional LNCs or OS LNCs. DiDLNCs and FP730-C18 LNCs were then mixed together (one mixture per LNC type) at a dilution of $1 / 200$ in PBS (Lonza) and kept at $37{ }^{\circ} \mathrm{C}$ in a moving plate for $24 \mathrm{~h}$. Emission spectra were recorded at $1 \mathrm{~h}, 3 \mathrm{~h}$, $5 \mathrm{~h}$ and $24 \mathrm{~h}$.

\subsection{In vivo biodistribution study}

The mice were injected in the tail vein with $200 \mu \mathrm{L}$ of FRET LNEs, FRET LNCS or FRET OS LNCs ( 6 animals per group) which corresponds for LNEs to $9.75 \mathrm{mg}$ total lipids, for conventional LNCs to $22.3 \mathrm{mg}$, and for OS LNCs to $23.4 \mathrm{mg}$. Fluorescence images were acquired at $1 \mathrm{~h}, 3 \mathrm{~h}$, $5 \mathrm{~h}$ and $24 \mathrm{~h}$ using the IVIS kinetic (Caliper Life Sciences, Hopkinton, USA). Three mice per group were sacrificed at $3 \mathrm{~h}$ and $24 \mathrm{~h}$ in order to image the organs and analyze the plasma. Image display and analysis were performed using the Living Image Software (Perkin-Elmer, Waltham, USA). For this purpose, the FRET signal was treated as a separate color, and deconvolution was performed using three different channels: DiD, FP730-C18, and FRET. It was checked on mixtures of DiD LNEs, FP730-C18 LNEs and FRET LNEs (or DiD LNCs, FP730-C18 LNCS, FRET LNCS) that image deconvolution allowed the discrimination of each type of nanoparticles in the mixture (Supporting information).

\section{Results and discussion}

\subsection{Design of a one-step stealth LNC (OS LNCS) process}

The first step of the study consisted of evaluating the feasibility of a direct stealth LNC formulation based on the phase inversion method. For this purpose, increasing concentrations of DSPE-mPEG ranging from $0.1 \%$ to $8.1 \%(\mathrm{w} / \mathrm{w})$ were added to the LNC mixture.

The physical characteristics of the obtained LNCs are summarized in Fig. 1. The formation of OS LNCs was successful up to a concentration of $6 \% \mathrm{w} / \mathrm{w}$ and the increasing addition of DSPE-mPEG led to a gradual increase in LNC diameter from $51.9 \pm 0.8 \mathrm{~nm}$ to $61.8 \pm 1.5 \mathrm{~nm}$. Beyond a concentration of $6 \%$, different size populations were observed suggesting an excess in DSPE-PEG addition. The resulting larger size of LNCs when DSPE-mPEG content is increased is consistent with previous reports on other PEGylated nanocarriers such as liposomes ( $+5 \mathrm{~nm},+$ $10 \mathrm{~nm})[25,26]$ and solid lipid nanoparticles $(+10 \mathrm{~nm})$ [27]. It was also the case with the post-insertion technique [24]. The size modification may have resulted from two factors. Firstly, the addition of PEGylated phospholipids into the shell composition may have altered its structure and thus the radius of curvature. Secondly, this DSPE-mPEG is composed of 45 PEG units which is much longer than the 15 units of the HS-PEG making the standard LNC formulation. These extended chains may have played a part in the increase in hydrodynamic diameter. For comparison, LNEs with PEG $_{40}$ stearate surfactants on their surface display a diameter of $58.0 \pm 2.1 \mathrm{~nm}$.

The DSPE-mPEG presence into the shell was further evidenced by a greater negative surface charge detected via the zeta measurement. This is typical of PEG coating onto nanoparticle surfaces as previously

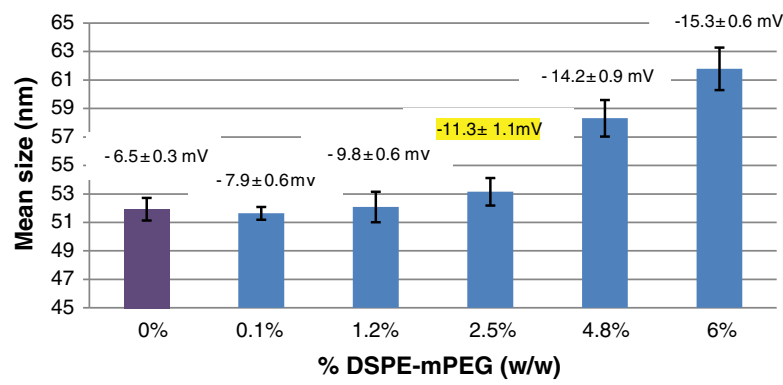

Fig. 1. Size and zeta potential of resulting one-step PEGylated-LNCs (OS LNCs) with increasing concentrations of DSPE-mPEG. 
described for other nanocarriers such as polymeric micelles [28], and liposomes [29]. This negative charge might be due to dipole interactions between PEG chain and water molecules as reported by Vonarbourg et al. [30] and to the negatively charged phosphate groups of the DSPEmPEG 2000 used in OS LNCs, concordant with the results reported in the literature [31]. Regarding the LNEs, the zeta potential was $-5.1 \pm$ $2.5 \mathrm{mV}$.

Despite the limited amount of DSPE-mPEG that can be grafted compared to that of the post-insertion technique [32], this one-step process provides a convenient and efficient method to obtain stealth LNCs. For the following studies, the DSPE-mPEG concentration was set at $4.8 \%$ $(\mathrm{w} / \mathrm{w})$.

\subsection{Physico-chemical stability studies}

In a previous study, a formulation of one-step stealth LNCs was attempted through the addition of a PEG 1500 based surfactant. However, the resulting LNCs showed increasing size over time [33]. Therefore, stability studies at $4{ }^{\circ} \mathrm{C}$ (storage condition) and $37{ }^{\circ} \mathrm{C}$ (physiological condition) were carried out over 3 weeks for OS LNCs in comparison to native LNCs and LNEs. Size and zeta potential were monitored at different time points and the results were plotted in Fig. 2. LNEs, conventional LNCs, and OS LNCS proved to be stable whatever the temperature condition. Interestingly, the DSPE-PEG grafting through the OS process did not alter the physico-chemical stability of the blank nanocapsules.

\section{Physical stability in plasma}

The drug carrier fate upon intravenous injection is mostly governed by plasma protein interactions. These interactions depend on the physicochemical properties of the carrier [34] and their nature affects the cell internalization process and the overall distribution throughout the body. In respect to this, the most investigated phenomenon is the opsonization effect referred to as the deposition of opsonins onto the carrier surface triggering carrier phagocytosis. This effect results in enhanced clearance of nanosystems. Besides altering trafficking of carriers, protein interactions can influence their stability which might induce early release of their payload. For a representative example of this consideration, the transfer of phospholipids from liposomes to high-density lipoproteins led to leakage of their contents [35]. The assessment of protein-nanoparticle interactions can be performed through nanoparticle incubation with plasma. This requires isolating
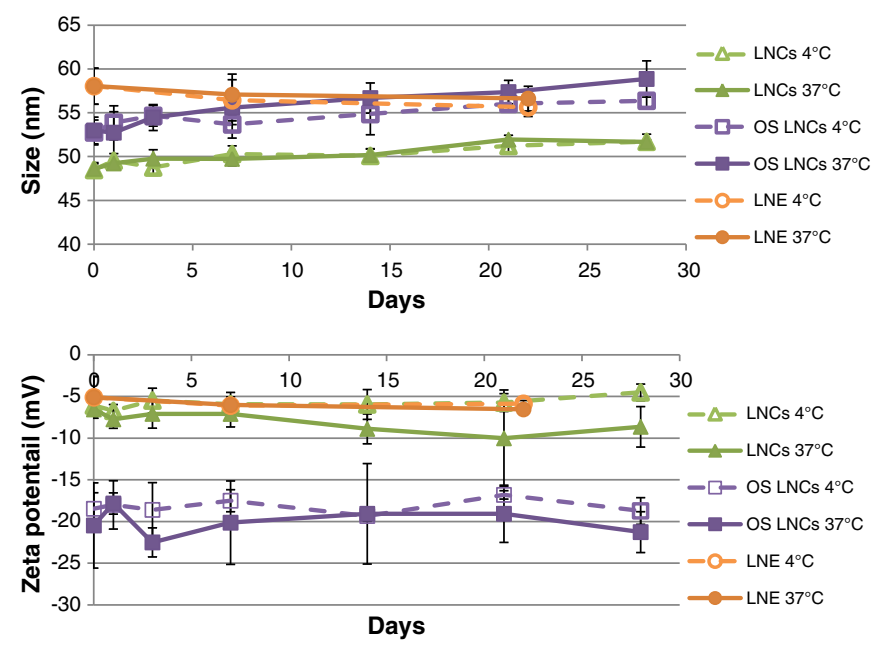

Fig. 2. Physico-chemical stabilities of conventional and OS LNCs at $4{ }^{\circ} \mathrm{C}$ and $37{ }^{\circ} \mathrm{C}$ over 28 days. the nanoparticle-protein complex from the excess proteins without disrupting or disturbing the complex, which is commonly achieved through a centrifugation step. However, centrifugation of lipid nanoparticles is challenging and still need further optimizations.

Consequently, we envisaged to preliminarily assess the potential interaction between lipid nanoparticles (LNCs, LNEs) and proteins, through the monitoring of the particle size during incubation with plasma. The rationale of this study is based on the consideration that if proteins interact with lipid nanoparticles through exchange or insertion in their structure, their hydrodynamic diameter would be affected.

Accordingly, LNEs, and conventional and OS LNCs were incubated with plasma to mimic in vivo conditions and their sizes were monitored at different time points (Fig. 3). Remarkably, LNEs and both kinds of LNCs showed stable sizes over $24 \mathrm{~h}$. After five incubation days, conventional LNCs displayed a larger hydrodynamic diameter whereas OS LNCs and LNEs preserved their size, emphasizing the role of long PEG chains in the LNC structure stability. Although further studies are required to determine the protein deposition on nanoparticle surfaces, this study showed for the first time that at least a part of the lipid nanoparticles (whatever the formulation) remained intact in plasma for $24 \mathrm{~h}$.

\section{Encapsulation stability in plasma}

Although the LNC structure did not seem to be affected by plasma proteins, their content might leak from the LNC core toward a more favorable protein medium. This leakage phenomenon from LNC has been recently pointed out by Bastiat et al. [36]. In an in vitro study, the authors investigated the impact of the fluorochrome nature on its encapsulation stability within the LNC after mixing the probe-loaded LNC suspension with oil. It was shown that lipophilic indocarbocyanines (i.e. DiO, Dil and DiD) anchored into the LNC shell through a long stearyl chain did not leak from the LNC whereas a transfer occurred from the cargo to the oil for other probes such as Nile red and 6-coumarin encapsulated in the nanoparticle core. This result highlights the possibility of content unloading without any LNC disassembly. This statement is a matter of concern since it might impair the drug delivery performance of a carrier. Consequently, the drug loaded lipid nanoparticle stability should be assessed in biologically relevant media. Again, the separation between the released component and the encapsulated one remains challenging.

FRET monitoring can cope with this issue by permitting to follow the release kinetics without the need of any separation step. Indeed, as a FRET signal can occur only when the two probes are in close proximity, any leak of one of them reduces the emitted signal. We recently demonstrated in a previous study that the FRET signal of DiI/DiD loaded LNEs and LNCs was maintained for at least $24 \mathrm{~h}$ when incubated at $37{ }^{\circ} \mathrm{C}$ in $1 \times$ PBS or BSA $(40 \mathrm{~g} / \mathrm{L})$, even in the presence of unloaded nanoparticles that could trigger lipid exchange [37]. It indicated that the good anchoring of the lipid dyes through the stearyl chain prevents dye leakage in

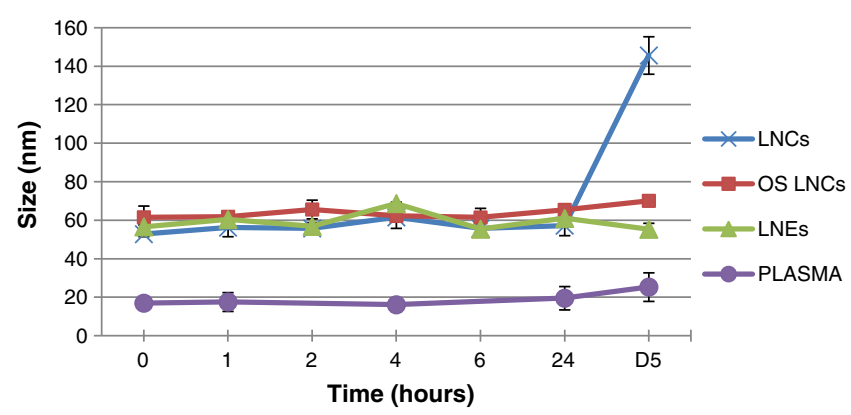

Fig. 3. LNC and LNE size stability in plasma. 
aqueous buffer. However, the use of the Dil/DiD FRET imaging pair (absorption/emission maxima: $\mathrm{Dil}=555 / 564 \mathrm{~nm}$ and $\mathrm{DiD}=651$ / $660 \mathrm{~nm}$ in the lipid particles) is limited for in vivo imaging, for which near infrared probes are required to limit light scattering and absorption by the tissues [37]. A common near infrared (NIR) probe used in the literature to design NIR FRET pairs is the DiR dye [22,23]. This fluorophore was discarded here since dye degradation has been shown to occur in a matter of weeks after its encapsulation in LNEs [6]. In the present study, we designed NIR FRET LNEs and LNCs through the encapsulation of the DiD/FP730-C18 pair (absorption/emission maxima: $\mathrm{DiD}=651 / 660 \mathrm{~nm}$ and FP730-C18 = 741/764 nm in the lipid nanoparticles - Förster radius $\mathrm{R}_{0}=6.6 \mathrm{~nm}$ for the DiD/FP730-C18 FRET pair, Supporting information). The hydrophilic FP730 dye was modified by an octadecyl (stearyl) C18 chain in order to make the encapsulation possible. Interestingly, the coencapsulation of DiD and FP730-C18 in LNEs leads to a high efficient energy transfer (90\% for DiD/FP730-C18 1:2.5, as further used in the plasma and in vivo experiments, see Supporting information). This transfer yield suggested that the fluorophores were located in a distance inferior to $6.6 \mathrm{~nm}$.

The stability of encapsulation of the DiD/FP730-C18 FRET pair in LNCs was evaluated during incubation in plasma through the FRET signal monitoring (Fig. 4). The fluorescence emission spectra for the conventional LNCs revealed a progressive reduction of the FRET signal intensity whereas the DiD donor signal increased over incubation time. As for the OS FRET LNCs, the FRET emission spectra appeared more stable. Accordingly, OS LNCs showed higher content stability in plasma over conventional LNCs. This might be owed to the steric barrier imposed by the PEGylated phospholipids which maintains a distance between proteins and LNCs subsequently preventing transfer to the biological medium, whereas intimate interactions can happen with conventional LNCs favoring content leakage. Additionally, these stealth LNCs seemed to exhibit higher stability of encapsulation than PEG-PDLLA micelles [38]. Indeed, these micelles had been shown to experience a fast release within 15 min after intravenous injection and further in vitro studies proved that $\alpha$-and $\beta$-globulins were responsible for this effect.
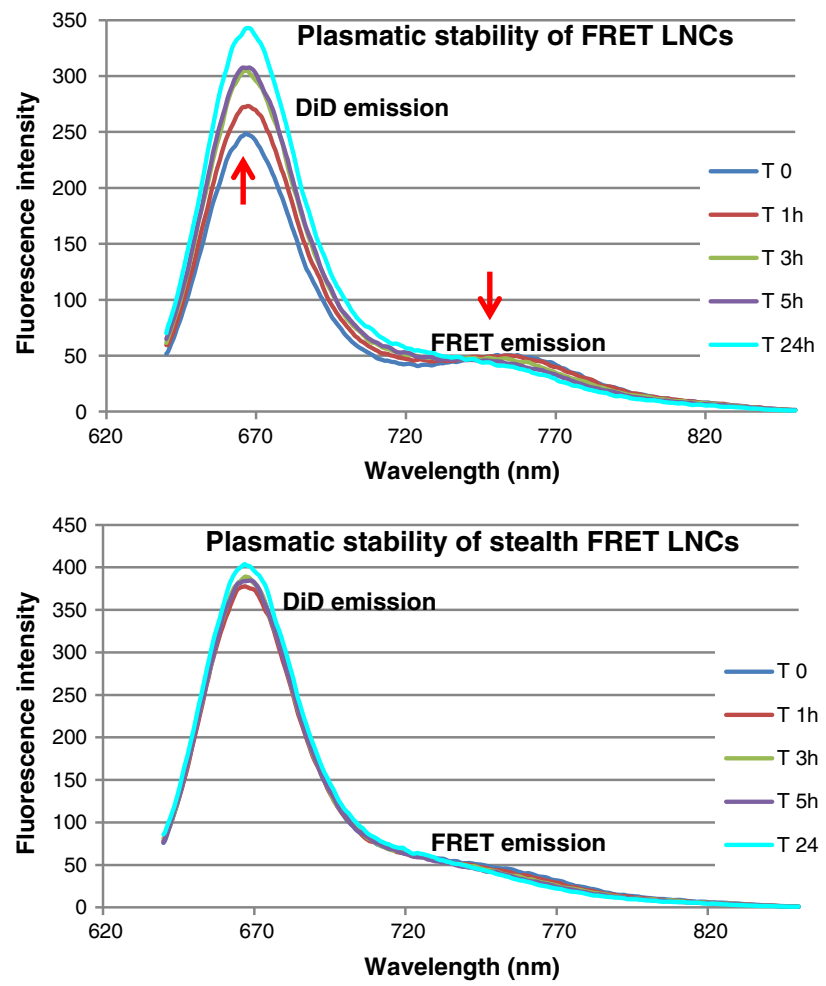

Fig. 4. Plasmatic stability of encapsulation in conventional LNCs and stealth LNCs.

\subsection{Exchange dynamics}

To further assess the good anchoring of both DiD and FP730-C18 in LNCs, we assessed the guest exchange dynamics in an aqueous medium based on a method developed by Jiwpanich et al. [39]. This method consists of encapsulating the donor and acceptor independently, then mixing DiD-LNCs and FP730-C18-LNCs and evaluating the occurrence of FRET signal implying transfer between the two carriers (Fig. 5). It is worth noting that the formulation of FRET LNCs is based on the capacity of pre-formulated DiD LNCs to incorporate the hydrophobic FP730-C18 probe upon overnight stirring. This highlights the ability of LNCs to act as an acceptor compartment.

At T0, no FRET could be observed since the distance between the two dye molecules was too far. Then, a slight exchange of guest molecules between DiD-LNCs and FP730-C18-LNCs occurred as indicated by the slight increase of the FRET signal. For both LNC types (conventional and stealth), a weak FRET signal was measured at $24 \mathrm{~h}$.

\subsection{FRET biodistribution}

The determination of nanoparticle distribution and accumulation in animal models is required before any application in humans. In this regard, various techniques had been applied in order to monitor the particle fate and some discrepancies could be observed depending on the tracer used. In a previous study, the co-labeling of LNEs with the DiD dye and two radiotracers led to different elimination phases in vivo depending on the imaging agent [40]. Similarly, a first attempt to monitor the LNC integrity was carried out through the co-labeling of the LNC shell with ${ }^{125} \mathrm{I}$ and LNC core with ${ }^{99 \mathrm{~m}}$ Technetium-oxine [40]. However, both radionuclides showed distinct distributions which did not permit to evaluate the LNC integrity. Since recently, increasing interest has been devoted to FRET imaging in living animals [22,38,23]. Cheng et al. injected Dil/DiD-loaded PLA-PEG micelles intravenously and studied their dissociation by confocal microscopy in the capillaries of the ear
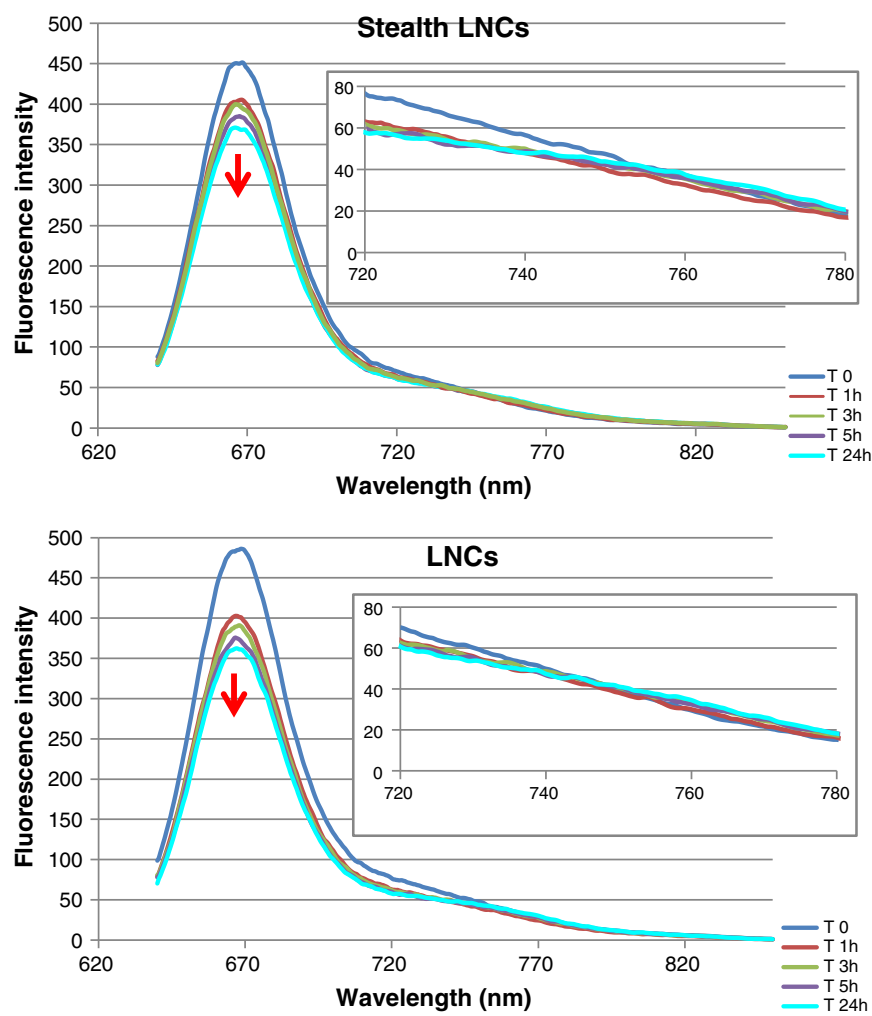

Fig. 5. Guest exchange dynamic studies of conventional LNCs and stealth LNCs. 
lobe of mice. This necessitated to place the ear under a coverslip but provided important results concerning the impact of blood components on the stability of these particles [38]. Wagh et al. designed PLGA-PEG polymeric nanoparticles encapsulating the DiD/DiR near-infrared FRET pair [22] and studied their integrity ex vivo on the mice organs, and in vivo using a capillary inserted in the mice. They noticed an important cross-talk and signal background present in "crude" FRET images.

In this work, we have intended to track the LNC and LNE integrity through a FRET technique. For that purpose, a NIR acceptor/donor pair (DiD and FP730-C18) was entrapped into the lipid nanoparticles and the resulting FRET signal was monitored and considered as the signal issued from the nanocargos. The biodistributions of FRET-nanoparticles were studied upon intravenous injection and compared. In vivo fluorescence images were acquired at $1 \mathrm{~h}, 3 \mathrm{~h}, 5 \mathrm{~h}$ and $24 \mathrm{~h}$ post injection and ex vivo organs were excised at $3 \mathrm{~h}$ and $24 \mathrm{~h}$ (Fig. 6). In order to separate the FRET contribution from that of the separated dyes, DiD and FP730C18, we used an image deconvolution method. This method is easy to implement, and gives a good discrimination between the FRET signal and the signal issued from a mixture of the separated dyes (Supporting information).

Regarding the conventional LNCs, one hour post-injection, a homogeneous FRET signal can be observed with a rather large distribution throughout the body highlighting the stability of the FRET LNC structure. At $3 \mathrm{~h}$, the FRET signal was less diffuse and the preferential sites for LNC accumulation were the liver, sternum, pelvic bones and backbone, suggesting a major uptake by Kupffer cells in the liver and macrophages in bone marrow. The retention in the liver was not surprising since it is the major nanoparticle elimination organ whereas the uptake in bones was more intriguing at first glance but has already been observed for silica nanoparticles [41]. Ex vivo organ images confirmed an intense FRET emission in the liver and a weaker signal in the spleen, another key organ responsible for nanoparticle clearance. Furthermore, DiD signal appeared in the intestine, skin (purple signal in the living animal) and lung whereas FP730-C18 arose from the kidney and the liver. In addition, the white plasma color was attributed to the superimposition of the FRET signal and both free probe signals. The presence of this FRET in blood provided evidence of the integrity of the particles $3 \mathrm{~h}$ post injection. However, the two distinct probe signals highlighted a partial release of the dyes either due to leakage from the LNCs or owed to the LNC disassembly.

Based on the fluorescence localization, the two probes showed distinct elimination pathways with a major biliary excretion for DiD and renal filtration associated with biliary excretion for FP730-C18. In consequence to this independent clearance pattern, the FRET signal undoubtedly did originate from the LNC rather than attributed to the forming of a plasmatic dye complex. The biodistribution observed at $5 \mathrm{~h}$ post injection was similar to that of $3 \mathrm{~h}$. At $24 \mathrm{~h}$, FRET from LNCs was mainly emitted from the intestine, indicative of a biliary excretion pathway. A signal
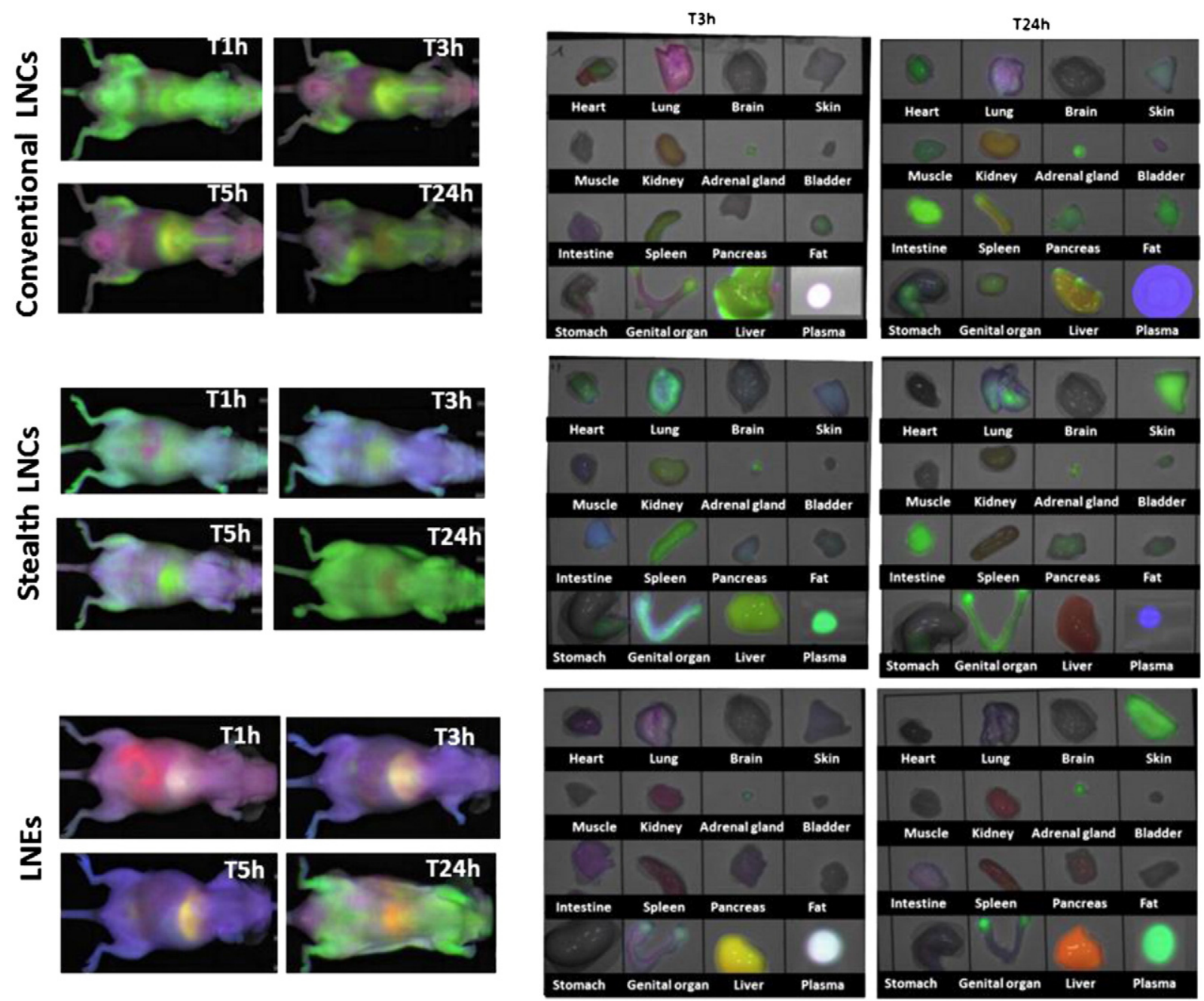

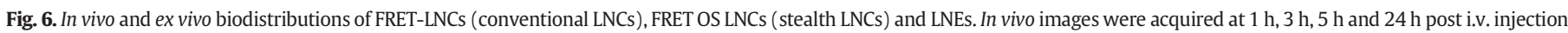
and ex vivo organ at $3 \mathrm{~h}$ and $24 \mathrm{~h}$ (green: FRET, blue: DiD, red: FP730-C18). 
could also be perceived from the heart and from the adrenal gland. In addition, DiD was mainly found in blood circulation whereas FP730C18 remained in the kidney and the liver.

In comparison to the conventional LNCs, the presence of the long PEG chains on the carrier surface of OS LNCs and LNEs markedly altered their biodistribution. Whereas an extensive FRET signal could be noticed at $1 \mathrm{~h}$ with conventional LNCs, such green emission could not be observed on the living animals for early times after injection of OS LNCs or LNEs, except for a small green area coming from the liver in the case of OS LNCs. The green fluorescence from the plasma at $3 \mathrm{~h}$ for OS LNCs, confirmed the integrity of most of the FRET OS LNCs circulating through the blood network without peripheral distribution. Additionally, it showed the extended circulation time of OS LNC over conventional LNCs. Besides blood, FRET appeared in the spleen, the liver and the lungs. For LNEs, the FRET signal observed in the blood was superimposed with the signals from FP730-C18 and DiD at $3 \mathrm{~h}$ (overall white color), and the FRET signal coming from the liver and ovaries was superimposed with the signal from DiD alone at $3 \mathrm{~h}$ (yellow-green color). The affinity for the ovary was already seen in our previous study [40]. As a matter of fact, it has been shown that various types of nanoparticles tend to accumulate in the ovary, most likely due to the EPR effect [42]. Although this requires further investigation regarding the toxicological aspect, this genital accumulation can also open new opportunities for an ovary targeted therapy.

As for conventional LNCs, a distinct signal from DiD alone was observed for OS LNCs with an accumulation in the lungs, the intestine and the skin. The skin signal is responsible for the general blue color of the living animal at $3 \mathrm{~h}$ and $5 \mathrm{~h}$ after injection of LNEs or OS LNCs, hampering deeper visualization of the still intact FRET nanoparticles. It has to be borne in mind that the fluorescence signal coming from the surface is far more intense than the signal coming from deeper tissue layers, principally because of the very important light scattering, already reducing by several factors the signal coming from a few mm under the skin. As DiD (blue) displays some affinity for the skin, it alters the aspect of the photos at early time points after injection and might mislead the interpretation. However, when considering organ biodistribution, the intense FRET signal in plasma at $3 \mathrm{~h}$ post-injection (stealth LNCs) proves the presence of the stealth nanoparticles in their intact form.

In contrast, FP730-C18 could hardly be noticed at $1 \mathrm{~h}, 3 \mathrm{~h}$ and $5 \mathrm{~h}$ after injection of OS LNCs, which might underlie a more stable FRET particle over conventional LNCs. By comparison, the FP730-C18 signal is higher after LNE injection, with distribution in the intestine, kidney, and to a less extent in the spleen and lungs, indicative of a biliary excretion associated with a lower extent renal filtration of this dye. It has to be noticed that lung and spleen FRET signals observed with LNEs were lower than for conventional and OS LNCs, and that no signal was observed in the bone marrow. This could indicate a lower propensity of LNEs for macrophage homing than for LNCs. On the other hand, FRET LNEs were less stable than OS LNCs, since at early time points fluorescence signals originating from DiD alone and FP730-C18 alone were stronger.

At $24 \mathrm{~h}$, OS LNCs were substantially located in the skin and partly in the intestine, likely due to biliary excretion. Conversely, FRET was no longer found in liver nor in spleen. As previously observed, DiD remained in the blood circulation whereas FP730-C18 was mostly, if not only, stuck in the liver. Similarly, FRET LNEs were present in the skin and ovaries, whereas liver signal was attributed to FP730-C18. In addition, a strong FRET signal was observed in plasma highlighting a markedly long circulation time. This, associated with a low macrophage uptake, accentuated great stealth properties, likely due to the long PEG chain coating.

The strong OS LNC and LNE accumulation in the skin is the major biodistribution difference in comparison to conventional ones. This cutaneous phenomenon has already been observed for PEGylated liposome [43]. It is worth noting that neither non-PEGylated liposomes [43] nor conventional LNCs achieved such a distribution. Moreover, the skin accumulation is not size-dependent since it had been observed whatever the size of the stealth liposomes from $80 \mathrm{~nm}$ to $240 \mathrm{~nm}$, with a slightly lesser extent for $240 \mathrm{~nm}$ liposomes. Consequently, long circulation property is the only requirement for the phenomenon to occur.

\section{Conclusion}

FRET imaging is a non-invasive and convenient technique allowing the integrity of nanoparticles as well as drug release kinetics to be monitored. Although this FRET technique is not a quantitative method, a suitable global picture of the lipid nanocarrier biodistribution could be acquired permitting to compare conventional LNCs to stealth lipid nanoparticles. In this work, a new stealth LNC formulation has been successfully developed through a one-step PEGylation process. The stability of encapsulation in LNCs turned out to be enhanced through the insertion of PEGylated phospholipids.

Regarding the biodistribution, stealth lipid nanoparticles (OS LNCS and LNEs) displayed extended blood circulation lifetime over conventional LNCs, as expected. The latter were eliminated progressively via the liver and the bone marrow macrophages while the long PEG chains modified the biodistribution pattern of the two other kinds of lipid nanoparticles. OS LNCs showed great structure stability several hours after intravenous injection with a subsequent accumulation in the intestine, ovaries and skin. Concerning the LNEs, on one hand, a burst release from a part of LNEs was observed in the early times with the emission of two signals from the free dyes. On the other hand, the part of LNEs remaining intact proved to be stable in plasma $24 \mathrm{~h}$ post injection.

Non-invasive FRET imaging has permitted to gain a greater insight into the behavior of our lipid nanoparticles revealing formulationdependent biodistribution and release kinetics.

\section{Acknowledgment}

This work was supported by the French National Research Agency (ANR, CALIF project no. ANR-08-NANO-006 and ANR Blanc Program Mecaferrol no. ANR-10-BLAN-706).

\section{Appendix A. Supplementary data}

Supplementary data to this article can be found online at http://dx. doi.org/10.1016/j.jconrel.2014.05.042.

\section{References}

[1] N.T. Huynh, C. Passirani, P. Saulnier, J.P. Benoit, Lipid nanocapsules: a new platform for nanomedicine, Int. J. Pharm. 379 (2009) 201-209, http://dx.doi.org/10.1016/j. ijpharm.2009.04.026.

[2] T. Delmas, H. Piraux, A.-C. Couffin, I. Texier, F. Vinet, P. Poulin, et al., How to prepare and stabilize very small nanoemulsions, Langmuir 27 (2011) 1683-1692, http://dx. doi.org/10.1021/la104221q.

[3] T. Delmas, A.-C. Couffin, P.A. Bayle, F. de Crécy, E. Neumann, F. Vinet, et al., Preparation and characterization of highly stable lipid nanoparticles with amorphous core of tuneable viscosity, J. Colloid Interface Sci. 360 (2011) 471-481, http://dx.doi.org/10. 1016/j.jcis.2011.04.080.

[4] M.B. Fabrice, P. Navarro, Lipid nanoparticle vectorization of indocyanine green improves fluorescence imaging for tumor diagnosis and lymph node resection, J. Biomed. Nanotechnol. 8 (2012) 730-741.

[5] M. Goutayer, S. Dufort, V. Josserand, A. Royère, E. Heinrich, F. Vinet, et al., Tumor targeting of functionalized lipid nanoparticles: assessment by in vivo fluorescence imaging, Eur. J. Pharm. Biopharm. 75 (2010) 137-147, http://dx.doi.org/10.1016/j. ejpb.2010.02.007.

[6] J. Gravier, F.P. Navarro, T. Delmas, F. Mittler, A.-C. Couffin, F. Vinet, et al., Lipidots: competitive organic alternative to quantum dots for in vivo fluorescence imaging, J. Biomed. Opt. 16 (2011) 096013, http://dx.doi.org/10.1117/1.3625405.

[7] A. Jacquart, M. Kéramidas, J. Vollaire, R. Boisgard, G. Pottier, E. Rustique, et al., Liplmage тм 815: novel dye-loaded lipid nanoparticles for long-term and sensitive in vivo nearinfrared fluorescence imaging, J. Biomed. Opt. 18 (2013) 101311, http://dx.doi.org/10. 1117/1.JBO.18.10.101311.

[8] F. Lacoeuille, F. Hindre, F. Moal, J. Roux, C. Passirani, O. Couturier, et al., In vivo evaluation of lipid nanocapsules as a promising colloidal carrier for paclitaxel, Int. J. Pharm. 344 (2007) 143-149, http://dx.doi.org/10.1016/j.ijpharm.2007.06.014.

[9] M.N. Khalid, P. Simard, D. Hoarau, A. Dragomir, J.-C. Leroux, Long circulating poly(ethylene glycol)-decorated lipid nanocapsules deliver docetaxel to solid tumors, Pharm. Res. 23 (2006) 752-758, http://dx.doi.org/10.1007/s11095-006-9662-5. 
[10] C. Vanpouille-Box, F. Lacoeuille, C. Belloche, N. Lepareur, L. Lemaire, J.-J. LeJeune, et al., Tumor eradication in rat glioma and bypass of immunosuppressive barriers using internal radiation with 188Re-lipid nanocapsules, Biomaterials 32 (2011) 6781-6790, http://dx.doi.org/10.1016/j.biomaterials.2011.05.067.

[11] A.-L. Laine, N.T. Huynh, A. Clavreul, J. Balzeau, J. Béjaud, A. Vessieres, et al., Brain tumour targeting strategies via coated ferrociphenol lipid nanocapsules, Eur. J. Pharm. Biopharm. 81 (2012) 690-693, http://dx.doi.org/10.1016/j.ejpb.2012.04.012.

[12] A. Barras, A. Mezzetti, A. Richard, S. Lazzaroni, S. Roux, P. Melnyk, et al., Formulation and characterization of polyphenol-loaded lipid nanocapsules, Int. J. Pharm. 379 (2009) 270-277, http://dx.doi.org/10.1016/j.ijpharm.2009.05.054.

[13] S. Vrignaud, N. Anton, P. Gayet, J.-P. Benoit, P. Saulnier, Reverse micelle-loaded lipid nanocarriers: a novel drug delivery system for the sustained release of doxorubicin hydrochloride, Eur. J. Pharm. Biopharm. 79 (2011) 197-204, http://dx.doi.org/10. 1016/j.ejpb.2011.02.015.

[14] M. Morille, T. Montier, P. Legras, N. Carmoy, P. Brodin, B. Pitard, et al., Longcirculating DNA lipid nanocapsules as new vector for passive tumor targeting, Biomaterials 31 (2010) 321-329, http://dx.doi.org/10.1016/j.biomaterials.2009.09.044.

[15] S. David, P. Resnier, A. Guillot, B. Pitard, J.-P. Benoit, C. Passirani, siRNA LNCs - a novel platform of lipid nanocapsules for systemic siRNA administration, Eur. J. Pharm. Biopharm. 81 (2012) 448-452, http://dx.doi.org/10.1016/j.ejpb.2012.02.010.

[16] S. Ballot, N. Noiret, F. Hindré, B. Denizot, E. Garin, H. Rajerison, et al., 99mTc/ 188Re-labelled lipid nanocapsules as promising radiotracers for imaging and therapy: formulation and biodistribution, Eur. J. Nucl. Med. Mol. Imaging 33 (2006) 602-607, http://dx.doi.org/10.1007/s00259-005-0007-0.

[17] N.T. Huynh, M. Morille, J. Bejaud, P. Legras, A. Vessieres, G. Jaouen, et al., Treatment of 9L gliosarcoma in rats by ferrociphenol-loaded lipid nanocapsules based on a passive targeting strategy via the EPR effect, Pharm. Res. 28 (2011) 3189-3198, http://dx.doi.org/10.1007/s11095-011-0501-y.

[18] J. Mérian, R. Boisgard, X. Decleves, B. Thezé, I. Texier, B. Tavitian, Synthetic lipid nanoparticles targeting steroid organs, J. Nucl. Med. 54 (2013) 1996-2003, http:// dx.doi.org/10.2967/jnumed.113.121657.

[19] E.A. Jares-Erijman, T.M. Jovin, FRET imaging, Nat. Biotechnol. 21 (2003) 1387-1395, http://dx.doi.org/10.1038/nbt896.

[20] J. Lu, S.C. Owen, M.S. Shoichet, Stability of self-assembled polymeric micelles in serum, Macromolecules 44 (2011) 6002-6008, http://dx.doi.org/10.1021/ma200675w.

[21] H. Chen, S. Kim, L. Li, S. Wang, K. Park, J.-X. Cheng, Release of hydrophobic molecules from polymer micelles into cell membranes revealed by Förster resonance energy transfer imaging, PNAS 105 (2008) 6596-6601, http://dx.doi.org/10.1073/pnas. 0707046105.

[22] A. Wagh, S.Y. Qian, B. Law, Development of biocompatible polymeric nanoparticles for in vivo NIR and FRET imaging, Bioconjug. Chem. 23 (2012) 981-992, http://dx. doi.org/10.1021/bc200637h.

[23] P. Zou, H. Chen, H.J. Paholak, D. Sun, Noninvasive fluorescence resonance energy transfer imaging of in vivo premature drug release from polymeric nanoparticles, Mol. Pharm. 10 (2013) 4185-4194, http://dx.doi.org/10.1021/mp4002393.

[24] T. Perrier, P. Saulnier, F. Fouchet, N. Lautram, J.-P. Benoît, Post-insertion into lipid nanocapsules (LNCs): from experimental aspects to mechanisms, Int. J. Pharm. 396 (2010) 204-209, http://dx.doi.org/10.1016/j.ijpharm.2010.06.019.

[25] R.-L. Hong, C.-J. Huang, Y.-L. Tseng, V.F. Pang, S.-T. Chen, J.-J. Liu, et al., Direct comparison of liposomal doxorubicin with or without polyethylene glycol coating in C-26 tumor-bearing mice: is surface coating with polyethylene glycol beneficial? Clin. Cancer Res. 5 (1999) 3645-3652.

[26] A.S. Abreu, E.M. Castanheira, M.-J.R. Queiroz, P.M. Ferreira, L.A. Vale-Silva, E. Pinto, Nanoliposomes for encapsulation and delivery of the potential antitumoral methyl 6-methoxy-3-(4-methoxyphenyl)-1H-indole-2-carboxylate, Nanoscale Res. Lett. 6 (2011) 482, http://dx.doi.org/10.1186/1556-276X-6-482.

[27] A. Fundarò, R. Cavalli, A. Bargoni, D. Vighetto, G.P. Zara, M.R. Gasco, Non-stealth and stealth solid lipid nanoparticles (SLN) carrying doxorubicin: pharmacokinetics and tissue distribution after i.v. administration to rats, Pharmacol. Res. 42 (2000) 337-343.
[28] J. Wang, D. Mongayt, V.P. Torchilin, Polymeric micelles for delivery of poorly soluble drugs: preparation and anticancer activity in vitro of paclitaxel incorporated into mixed micelles based on poly(ethylene glycol)-lipid conjugate and positively charged lipids, J. Drug Target. 13 (2005) 73-80, http://dx.doi.org/10.1080/ 10611860400011935

[29] W.L.J. Hinrichs, F.A. Manceñido, N.N. Sanders, K. Braeckmans, S.C. De Smedt, J Demeester, et al., The choice of a suitable oligosaccharide to prevent aggregation of PEGylated nanoparticles during freeze thawing and freeze drying, Int. J. Pharm. 311 (2006) 237-244, http://dx.doi.org/10.1016/j.ijpharm.2005.12.032.

[30] A. Vonarbourg, P. Saulnier, C. Passirani, J.-P. Benoit, Electrokinetic properties of noncharged lipid nanocapsules: influence of the dipolar distribution at the interface, Electrophoresis 26 (2005) 2066-2075, http://dx.doi.org/10.1002/elps.200410145.

[31] T. Yang, F.-D. Cui, M.-K. Choi, J.-W. Cho, S.-J. Chung, C.-K. Shim, et al., Enhanced solubility and stability of PEGylated liposomal paclitaxel: in vitro and in vivo evaluation, Int. J. Pharm. 338 (2007) 317-326, http://dx.doi.org/10.1016/j.ijpharm. 2007.02.011.

[32] D. Hoarau, P. Delmas, S. David, E. Roux, J.-C. Leroux, Novel long-circulating lipid nanocapsules, Pharm. Res. 21 (2004) 1783-1789.

[33] S. Hirsjärvi, S. Dufort, J. Gravier, I. Texier, Q. Yan, J. Bibette, et al., Influence of size, surface coating and fine chemical composition on the in vitro reactivity and in vivo biodistribution of lipid nanocapsules versus lipid nanoemulsions in cancer models, Nanomedicine 9 (2013) 375-387, http://dx.doi.org/10.1016/j.nano.2012.08.005.

[34] P. Aggarwal, J.B. Hall, C.B. McLeland, M.A. Dobrovolskaia, S.E. McNeil, Nanoparticle interaction with plasma proteins as it relates to particle biodistribution, biocompatibility and therapeutic efficacy, Adv. Drug Deliv. Rev. 61 (2009) 428-437, http://dx. doi.org/10.1016/j.addr.2009.03.009.

[35] T.M. Allen, A study of phospholipid interactions between high-density lipoproteins and small unilamellar vesicles, Biochim. Biophys. Acta Biomembr. 640 (1981) 385-397, http://dx.doi.org/10.1016/0005-2736(81)90464-8.

[36] G. Bastiat, C.O. Pritz, C. Roider, F. Fouchet, E. Lignières, A. Jesacher, et al., A new too to ensure the fluorescent dye labeling stability of nanocarriers: a real challenge for fluorescence imaging, J. Control. Release 170 (2013) 334-342, http://dx.doi.org/10. 1016/j.jconrel.2013.06.014.

[37] J. Gravier, L. Sancey, S. Hirsjärvi, E. Heinrich, C. Passirani, J.-P. Benoit, et al., FRET imaging approaches for in vitro and in vivo characterization of synthetic lipid nanoparticles, 2014. (submitted for publication).

[38] H. Chen, S. Kim, W. He, H. Wang, P.S. Low, K. Park, et al., Fast release of lipophilic agents from circulating PEG-PDLLA micelles revealed by in vivo forster resonance energy transfer imaging, Langmuir 24 (2008) 5213-5217, http://dx.doi.org/10. 1021/la703570m

[39] S. Jiwpanich, J.-H. Ryu, S. Bickerton, S. Thayumanavan, Non-covalent encapsulation stabilities in supramolecular nanoassemblies, J. Am. Chem. Soc. 132 (2010) 10683-10685, http://dx.doi.org/10.1021/ja105059g.

[40] A. Cahouet, B. Denizot, F. Hindré, C. Passirani, B. Heurtault, M. Moreau, et al. Biodistribution of dual radiolabeled lipidic nanocapsules in the rat using scintigraphy and $\gamma$ counting, Int. J. Pharm. 242 (2002) 367-371, http://dx.doi.org/10.1016/ S0378-5173(02)00218-1.

[41] H. Chen, Z. Zhen, W. Tang, T. Todd, Y.-J. Chuang, L. Wang, et al., Label-free luminescent mesoporous silica nanoparticles for imaging and drug delivery, Theranostics 3 (2013) 650-657, http://dx.doi.org/10.7150/thno.6668.

[42] A. Schädlich, S. Hoffmann, T. Mueller, H. Caysa, C. Rose, A. Göpferich, et al., Accumulation of nanocarriers in the ovary: a neglected toxicity risk? J. Control. Release 160 (2012) 105-112, http://dx.doi.org/10.1016/j.jconrel.2012.02.012.

[43] G.J.R. Charrois, T.M. Allen, Rate of biodistribution of STEALTH(r) liposomes to tumor and skin: influence of liposome diameter and implications for toxicity and therapeutic activity, Biochim. Biophys. Acta Biomembr. 1609 (2003) 102-108, http://dx.doi. org/10.1016/S0005-2736(02)00661-2. 